\title{
Die Verwendung des farbigen Cephalosporin- derivates PADAC zur enzymatischen Bestimmung von Cefotaxim in Serum- und Harnproben
}

\author{
G. Seibert, M. Limbert
}

Hoechst, Frankfurt/M.

\section{Zusammentassung:}

Eine einfache, enzymatische Methode zur Bestimmung des Cephalosporinderivates Cefotaxim unter Verwendung des farbigen Cephalosporinderivates PADAC wird beschrieben. Die Methode ist relativ spezifisch und ermöglicht eine schnelle und genaue Bestimmung des Antibiotikums in Serum und Harn ohne Vorbehandlung der Proben. Die Nachweisempfindlichkeit ist mit der von anderen Methoden zur Cefotaximbestimmung vergleichbar.

\author{
Schlüsse/wörter: \\ Bestimmung von Cefotaxim - PADAC
}

\section{Summary:}

An enzymatic method using the coloured Cephalosporin derivative PADAC is described for the determination of the cephalosporin derivative, cefotaxim, in serum and urine. The method is simple, relatively specific, rapid and accurate, and does not involve pretreatment of samples. The sensitivity is similar to that of other methods of cefotaxim assay.

Keywords:

Estimation of Cefotaxime - PADAC

\section{Einleitung}

Das Breitspektrumantibiotikum Cefotaxim (Claforan ${ }^{*}$ ) wird seit seiner Ausbietung in Deutschland bei bakteriellen Infektionen immer häufiger als Antibiotikum der ersten Wahl eingesetzt. Bei den zahlreichen pharmakokinetischen Untersuchungen mit diesem-Cephalosporin wurden meist die klassischen Bestimmungsmethoden, wie der mikrobiologische-Agardiffusionstest oder die Hochdruck-FlüssigkeitsChromatographie, eingesetzt $(1-3)$. Diese Methoden verursachen jedoch oft Schwierigkeiten, da in vielen Labors diese aufwendigen Verfahren nicht routinemäßig durchgeführt werden. Wir beschäftigten uns deshalb mit der Entwicklung einer enzymatischen Methode unter Verwendung eines chromogenen Cephalosporinderivates. Die Bestimmung ist einfach durchzuführen. Das verwendete Enzym, eine $\beta$-Laktamase, ist leicht zugänglich. Als Gerät ist lediglich ein einfaches Photometer, das im Bereich des sichtbaren Lichtes arbeitet, erforderlich.

Die Bestimmungsmethode beruht auf der kompetitiven Hemmung einer $\beta$-Laktamase durch ein $\beta$-Laktam-Antibiotikum (4-6). Das farbige Cephalosporinderivat (Abb. 1) 7 - (Thienyl-2 - Acetamido) - 3 - [2 - (4 - N,N - DimethylaminoPhenyl-Aco) - Pyridinium-Methyl] - 3-Cephem-4-Carboxylat (PADAC ${ }^{*}$ (7), wird von der $\beta$-Laktamase aus Enterobacter cloacae P99 sehr schnell hydrolysiert. Dabei schlägt die Farbe dieses Cephalosporins von rot nach gelb um. Der Farbum- schlag ist im Photometer bei $550 \mathrm{~nm}$ Wellenlänge leicht zu verfolgen. Die Geschwindigkeit, mit der das farbige Substrat durch das Enzym umgesetzt wird, kann verringert werden, wenn ein zweites Cephalosporin, das als kompetitiver Inhibitor wirkt, der Lösung zugesetzt wird. Als ein solcher Inhibitor eignet sich hervorragend Cefotaxim. Der Grad der Hemmung des Umsatzes von PADAC ist mit der Menge Cefotaxim in der zu analysierenden Probe korreliert. Im Gegensatz zu einer ebenfalls praktizierten Methode (8) mit einem nicht farbigen Cephalosporin erlaubt die Verwendung des farbigen Cephalosporins PADAC die Verwendung eines Photometers im sichtbaren Licht.<smiles>CN(C)c1ccc(N=Nc2cccc[n+]2CC2CSC3[C@H](NC(=O)[O-])C(=O)N3[C@H]2C(=O)[O-])cc1</smiles>

Abb. 1: Cephalosporinderivat PADAC 7-(Thienyl-2-Acetamido)-3-[2-(4-N.N-Dimethylamino-Phenyl-Azo)-Pyridinium-Methyl]-3-Cephem-4-Carboxylat 


\section{Material und Methoden}

Das farbige Cephalosporinderivat PADAC wurde von Calbiochem (Gießen) bezogen. Cefotaxim und Desacetyl-Cefotaxim stammten aus der Hoechst AG, Frankfurt/M.

\section{Enzympräparation}

Zur Enzympräparation wurde Enterobacter cloacae P99 in belüftetem Müller-Hinton-Medium bei $37^{\circ} \mathrm{C}$ kultiviert. Nach Abzentrifugation der Zellen wurde das Sediment in $0,1 \mathrm{~m}$ Phosphatpuffer ( $\mathrm{pH} \mathrm{7)} \mathrm{aufgenommen} \mathrm{und} \mathrm{durch} \mathrm{Ultraschall-}$ behandlung aufgeschlossen. Zelltrümmer wurden sodann bei $15000 \times \mathrm{g}$ in $20 \mathrm{~min}$ abzentrifugiert. Der enzymhaltige Überstand wurde durch Gelfiltration in Sephacryl S 200 (Deutsche Pharmacia, Freiburg) gereinigt. Die enzymatische Aktivität enthaltenden Fraktionen wurden bei $-20^{\circ} \mathrm{C}$ aufbewahrt und als Enzymlösung ohne weitere Reinigung verwendet. Die Aktivität des Enzyms war 38 mkatal pro kg Protein bei Verwendung von Cephaloridin als Substrat.

\section{Testansatz}

PADAC ist in Wasser nur sehr schwer löslich. Es wurden deshalb $2 \mathrm{mg}$ PADAC in 0,2 $\mathrm{ml}$ DMSO vorgelöst und mit Phosphatpuffer pH-Wert 7 auf $10 \mathrm{ml}$ aufgefüllt. Zu einer Be-

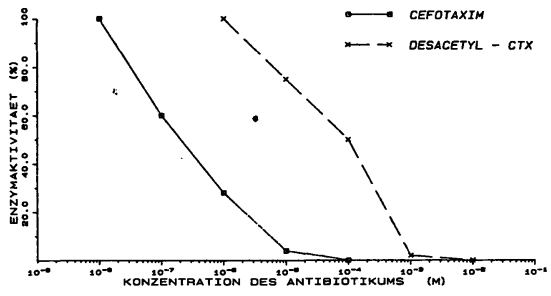

Abb. 2: Hemmung des enzymatischen Abbaus von PADAC durch Zusatz von Cefotaxim (-) und Desacetyl-Cefotaxim $(x--x)$

Tab. 1: Zusatz eines internen Standards von Cefotaxim zu einer Serumprobe

\begin{tabular}{lll}
\hline $\begin{array}{l}\text { Menge Cefotaxim } \\
\text { enzymatisch be- } \\
\text { stimmt in der Probe } \\
\text { pro ml Serum } \\
\mu \mathrm{g}\end{array}$ & $\begin{array}{l}\text { Menge Cefotaxim } \\
\text { als interner Stan: } \\
\text { dard zugesetzt } \\
\text { pro ml Serum } \\
\mu \mathrm{g}\end{array}$ & $\begin{array}{l}\text { Gesamtmenge } \\
\text { Cefotaxim enzymatisch } \\
\text { bestimmt pro ml } \\
\text { Serum } \\
\mu \mathrm{g}\end{array}$ \\
\hline $98 \pm 4,8$ & 10 & $112 \pm 6,8$ \\
$98 \pm 4,8$ & 50 & $159 \pm 7,4$ \\
$98 \pm 4,8$ & 75 & $179 \pm 9,3$ \\
$98 \pm 4,8$ & 100 & $216 \pm 4,5$ \\
\hline
\end{tabular}

stimmung wurden $0,2 \mathrm{ml}$ dieser PADAC-Lösung mit $1,7 \mathrm{ml}$ Phosphatpuffer pH 7.0 (0,1 m) gemischt und $0.1 \mathrm{ml}$ der zu analysierenden Lösung zugefügt. Die Reaktion wurde durch Zugabe von $0.1 \mathrm{ml}$ der $\beta$-Laktamase-Lösung gestartet. Die Extinktion dieser Lösung wurde sodann über $100 \mathrm{sec}$ bei $20^{\circ} \mathrm{C}$ im Photometer verfolgt. Auf diese Weise wurde eine Standardkurve für Cefotaxim in Wasser, und nach Zusatz bekannter Mengen Cefotaxim in Serum oder Harn aufgenommen. Entsprechende Versuche wurden mit Desacetylcefotaxim durchgeführt, um eine Fehlbestimmung durch diesen Metaboliten auszuschließen.

\section{Ergebnisse und Diskussion}

Wie Abb. 2 zeigt, eignet sich Cefotaxim sehr gut als kompetitiver Inhibitor für die Umsetzung von PADAC mit der $\beta$-Laktamase aus Enterobacter P99. Bei einer Konzentration $>10^{-8} \mathrm{~mol} / \mathrm{l} \mathrm{im}$ Reaktionsansatz ist eine Hemmung der enzymatischen Reaktion zu messen. Bei einer Konzentration von $10^{-4} \mathrm{~mol} / \mathrm{l}$ ist kein Umsatz des PADAC mehr festzustellen. Abb. 2 zeigt außerdem die Hemmkurve für Desacetylcefotaxim, dem Hauptmetaboliten von Cefotaxim. Mit dieser Substanz ist bei einer Konzentration von $10^{-6} \mathrm{~mol} / \mathrm{I}$ noch keine Störung der enzymatischen Reaktion des P99-Enzyms mit PADAC zu messen. Erst bei Konzentrationen $>10^{-6} \mathrm{~mol} / \mathrm{I}$ wirkt auch Desa-

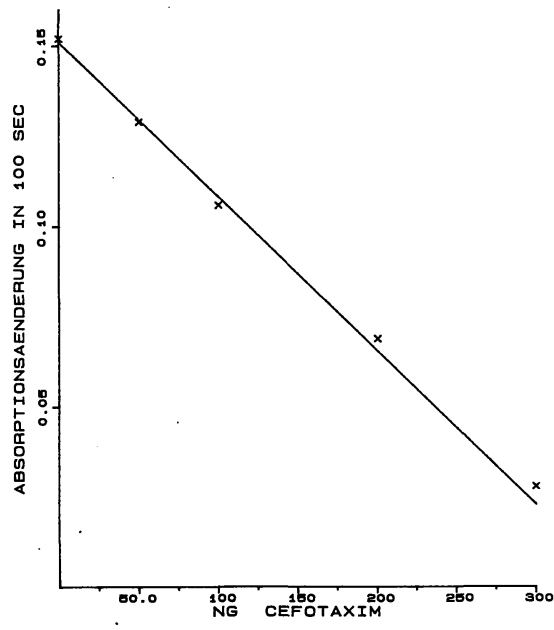

Abb. 3: Standardkurve zur Bestimmung von Cefotaxim in Serum.

$x$-Achse: Konzentration des Cefotaxim in der Küvette (s. Material und Methoden).

y-Achse: Absorptionsänderung in den ersten 100 sec der Reaktion.

Bei Zugabe des Cefotaxim in $0,1 \mathrm{ml}$ Harn statt in Serum ändert sich der Verlauf der Standardkurve nicht. 
cetylcefotaxim als Inhibitor. Das bedeutet, daß in einem Gemisch von Muttersubstanz und Metabolit Desacetyicefotaxim erst bei einer Konzentration, die größer als $10^{-6} \mathrm{~mol} / \mathrm{l}$ ist, als Inhibitor in Frage kommt, während Cefotaxim bereits bei höheren Verdünnungen als Inhibitor in Frage kommt. Wie Abb. 3 zeigt, ist unter den gewählten Testbedingungen die Aufnahme einer Standardkurve für Cefotaxim in einen Bereich bis $300 \mathrm{ng}$ im Ansatz möglich. Hier ist eine nahezu lineare Abhängigkeit zwischen zugesetzter Menge Cefotaxim und der Absorptionsänderung während der ersten 100 Sekunden Reaktionszeit gegeben. Die Standardkurve zeigt dển gleichen Verlauf beim Zusatz von Cefotaxim in Serum, Harn oder Pufferlösung. Die Bindung von Cefotaxim an Serumproteine hat keinen Einfluß auf den Nachweis von Cefotaxim in dieser Bestimmungsmethode. Die mittlere Abweichung der Standardwerte lag stets bei maximal $7 \%$.

Bei der Verwendung von klinischen Proben, also Cefotaxim in Serum, Harn oder anderen Körperflüssigkeiten, ist die Methode sehr gut verwendbar. Sie liefert die Versuchsergebnisse in kürzester Zeit. Wie Tab. 1 zeigt, ist bei Zugabe eines internen Standards zu Serum- oder Harnproben aus klinischen Versuchen stets ein Anstieg des Cefotaximgehaltes in Höhe des zugesetzten Antibiotikums zu messen. Das Verfahren stellt somit eine sehr einfache und schnelle Möglichkeit zur Bestimmung von Cefotaxim dar.

Eine Schwierigkeit könnte die Präparation und Aufbewahrung des Enzyms darstellen. Aber auch das sollte unter der Bedingung der klinischen Routine kein allzu großes Problem darstellen. Selbst wenn die Reinigung des Enzyms durch Gelchromatographie nicht möglich ist, sind durch Verwendung eines rohen Lysates des $\beta$-Laktamase bildenden Keimes gute Ergebnisse zu erzielen. In einen solchen Rohextrakt ist allerdings nach unserer Erfahrung die Stabilität des Enzyms etwas geringer. Gereinigte Enzymfraktionen aus Enterobacter P99 zeigen bei Aufbewahrung bei $-20^{\circ} \mathrm{C}$ über mehrere Wochen keinen signifikanten Aktivitätsverlust.

Neben Cefotaxim kann man mit einer ähnlichen Methode auch andere $\beta$-Laktam-Antibiotika bestimmen, die sich als kompetitive Inhibitoren für $\beta$-Laktamasen eignen. Allerdings müssen dabei Enzym und Substratkonzentration jeweils optimiert werden.

Schrifttum:

1. BERGAN, T., SOLBERG, R.: Assay of cefotaxime by high-pressure-liquid chromatography. Chemotherapy 27. 155-165 (1981)

2. FILLASTRE, J. P., LEROY, A., HUMBERT, G., GOOIN, M.: Pharmacokinetics of Cefotaxime in subjects with normal and impaired renal function. $J$. Antimicrob. Chemotherapy 6, Suppl. A, 103-111 (1980).

3. KEES, F., STREHL, E., SEEGER, K., SEIDEL, G., DOMINIAH, P., GROBECKER, H.: Comparative determination of cefotaxime and desacetyl-cefotaxime in serum and bile by bioassay and high-performance liquid chromatography. Arzneim. Forsch./Drug Res. 31 $362-365$ (1981)

4. COLE, M., ELSON, S., FULLBROOK, P. D.: Inhibition of the $\beta$-lactamases of E, coli and Klebsiella aerogenes by semi-synthetic penicillins. Biochem. J. 127, 295-308 (1972). 5. O'CALLAGHAN, C. H., MUGGLETON, P. W., KIRBY, S. M., RYAN, D. M.: Inhibition of B-lactamase decomposition of cephaloridin and cephalothin by other cephalosporins. Antimicrob. Ag. Chemother $337-343,1966$

6. O'CALLAGHAN, C. H.. MORRIS, A.: Inhibition of $\beta$-lactamases by $\beta$-lactarn antibiotics Antimicrob. Ag. Chemother. 2. 442-448 (1972)

7. SCHINDLER, P., HUBER, G.: Use of PADAC, a novel chromogenic $\beta$-lactamase substrate, for the detection of $\beta$-lactamase producing organisms and assay of $\beta$-lactamase inhibitors/inactivators "Proceedings on enzyme inhibition". Symposion in Basel 1980, im Druck.

8. SEIBERT, G., BIEBACH, A.: Eine enzymatische Methode zur Bestimmung von Cefotaxim in Serum- und Harnproben. J. Clin. Chem. Clin. Biochem. 19, 279-281 (1981).

Anschrift der Verfasser:

PD Dr. Gerhard Seibert

Dr. Michael Limbert

Hoechst AG, Abt. Chemotherapie

D-6230 Frankfurt/M. 80 